\title{
Envelhecimento cutâneo da pele: relação entre o excesso de carboidratos e a Reação de Maillard na formação de produtos de glicação avançada (AGES)
}

O presente trabalho teve por objetivo, através de uma revisão bibliográfica, mostrar a relação do excesso de carboidratose a Reação de Maillard no organismo, associado a formação de produtos finais da glicação avançada (advanced glycation end-products, AGEs), no processo de envelhecimento cutâneo da pele. O processo de envelhecimento altera a estrutura e a função dos órgãos, assim como a pele. Além de que, a mudança nos hábitos alimentares da população, que consomem, em sua maioria, produtos industrializados, com preferência alimentos ricos em carboidratos e gorduras, contribui para um envelhecimento precoce. Logo, uma alimentação adequada, rica em frutas e verduras, proporciona um efeito antioxidante, de proteção a estrutura da pele e a manutenção da pele rejuvenescida.

Palavras-chave: Envelhecimento Cutâneo; Pele; Glicação Avançada; Reação de Maillard; Antioxidantes.

\section{Skin aging: relationship between carbohydrate excess and Maillard's Reaction in advanced glycation products (AGES)}

The objective of the present work was to review the relationship between excess carbohydrate and Maillard Reaction in the body, associated with the formation of advanced glycation end-products (AGEs), in the aging skin. The aging process alters the structure and function of organs as well as the skin. In addition, the change in the population's eating habits, which consume, for the most part, industrialized products, with preference for foods rich in carbohydrates and fats, contributes to an early aging. Therefore, an adequate diet, rich in fruits and vegetables, provides an antioxidant effect, protecting the structure of the skin and maintaining rejuvenated skin.

Keywords: Skin Aging; Skin; Advanced Glycation; Maillard Reaction; Antioxidants.

Topic: Bioquímica

Reviewed anonymously in the process of blind peer
Received: $12 / 11 / 2017$

Approved: $24 / 01 / 2018$ 


\section{INTRODUÇÃO}

O envelhecimento é uma dos temas mais relevantes da medicina atual, visto que houve um aumento da expectativa de vida da população e, concomitantemente, a procura de novas formas para retardá-lo (SCHALKA et al., 2016). Quando falamos em envelhecimento cutâneo, reconhecemos, primeiramente, o sol como o fator ambiental mais relevante, através da ação oxidativa das radiações solares (os raios UVA), mas a ciência tem procurado avaliar outros fatores ambientais além da radiação solar, que possam contribuir no processo de aceleração do envelhecimento cutâneo extrínseco (SCHALKA et al., 2016).

A vida moderna, em especial, nos centros urbanos, sugere um estilo de vida bem diferente do passado, com exposição a fatores ambientais novos como a poluição, tabaco, alimentação inadequada, sono irregular, estresse emocional e ritmo de vida acelerado (SCHALKA et al., 2016; SCHALKA et al., 2017). Desse modo observa-se que nas últimas décadas, houve uma mudança de hábitos alimentares na população brasileira, levando a uma inadequação alimentar, por meio do aumento no consumo dos alimentos processados e industrializados, tipo fast food, com alto teor de carboidratos e gorduras, e teor reduzido de vitaminas e minerais, relacionadas ao avanço do número de pessoas que se alimentam fora de casa (SHIBAO, et al., 2011; SCHALKA et al,2017).

A maioria desses alimentos consumidos passam por processamento térmico, o que garante a segurança microbiológica, inativação de algumas enzimas, degradação de substâncias tóxicas e substâncias responsáveis pelo aroma, cor e sabor, melhorando a palatabilidade do alimento a ser consumido (SHIBAO et al., 2011). Essas substâncias são características da Reação de Maillard (RM) e são denominadas de forma genérica, de Produtos da Reação de Maillard (BARBOSA et al., 2008; BARBOSA et al., 2009).

Um dos produtos derivados da RM são os produtos da glicação avançada, as AGEs, estes relacionados de forma direta ao envelhecimento, visto que são substâncias de efeitos pró-oxidativo e pró-inflamatórios (BARBOSA et al., 2016; GOLDBERG et al., 2004; LAPOLLA et al., 2004). Assim a relação entre o envelhecimento cutâneo e a dieta inadequada pode ser explicada através da glicação entre açúcares reduzidos como a glicose e proteínas como o colágeno (SCHALKA et al.,2016; GOLDBERG et al., 2004). Assim, o objetivo deste artigo é fazer um revisão de literatura mostrando a relação do excesso de carboidratos e a Reação de Maillard no organismo, associado a formação de produtos finais da glicação avançada e os AGEs, no processo de envelhecimento cutâneo precoce da pele.

\section{METODOLOGIA}

Realizou-se uma revisão do conhecimento disponível na literatura científica, de artigos publicados entre 1999 e 2017, nas bases de dados Scielo (Scientific Eletronic Library Online), Medline (Medical Literature Analysis and Retrieval Systen Online), LILACS (Literatura Latino-Americana e do Caribe em Ciências da Saúde), Elsevier e google acadêmico, sendo que foram tomados para consulta às bases de dados os seguintes descritores: produtos finais de glicação avançada; nutrição e estética; envelhecimento cutâneo da pele; reação de Maillard; e antioxidantes, nas línguas portuguesa e inglesa. 


\section{DISCUSSÃO TEÓRICA}

Pele

A pele, maior órgão do corpo humano, é um órgão de revestimento bastante complexo e heterogêneo, sendo constituída fundamentalmente de três camadas de tecidos: uma superior, a epiderme; uma intermediária, a derme; e uma profunda, a hipoderme (TESTON et al., 2010; BENTO, 2015). Sendo que a pele apresenta funções de estética, proteção, nutrição, perspiração, transpiração, pigmentação, queratogênese, termorregulação, defesa e absorção da radiação solar(UV), síntese de vitamina $D$ e eliminação de substâncias químicas (BENTO, 2015; AGUIAR et al., 2017).

O colágeno é a proteína mais abundante do organismo, sendo o colágeno tipo l o mais predominante. As principais células da derme, os fibroblastos, sintetizam colágeno e elastina, e a degradação destes dois produtos é que acaba provocando as rugas e perda da elasticidade da pele (TESTON et al., 2010). Durante a infância o colágeno é mais abundante e na puberdade a sua produção é diminuída, por volta dos 20-30 anos de idade a produção do colágeno é estabilizada, sendo na velhice interrompida (TESTON et al., 2010).

Em torno dos 20 anos de idade, a pele jovem, na maioria das vezes, apresenta-se uniforme quanto à textura, cor, firmeza, ausência de rugas e manchas, mas com o envelhecimento, a partir dos 40 anos de idade, ocorre uma redução das fibras de colágeno e no nível de estrogênios, fazendo com que a pele se torne mais fina, manchada e sensível, levando ao surgimentos de rugas e células mortas, estas se depositando e acumulando na superfície (BATISTELA et al., 2007; TESTON et al., 2010). Assim a formação de rugas, pele mais áspera, elasticidade e firmeza da pele reduzida são os sinais mais expressivos do avanço da idade biológica (BATISTELA et al., 2007; HIRATA et al., 2004).

\section{Envelhecimento cutâneo}

O envelhecimento cutâneo, é um processo esperado, progressivo, dinâmico e irreversível, resultante de um declínio das funções fisiológicas do tecido cutâneo, tanto no nível dérmico quanto no epidérmico (BENTO, 2015; AGUIAR et al., 2017; ADDOR, 2015). É um processo influenciado pela genética, fatores comportamentais e ambientais, os quais envolvem uma sequência de alterações que ocorrem ao longo da vida e levam a perda das funções de vários órgãos (BENTO, 2015; TRAMONTINO, 2009).

Sendo assim, podem ser identificados dois tipos de causas do envelhecimento cutâneo: o cronológico ou intrínseco, caracterizado pelo desgaste natural do organismo, resultante da passagem do tempo e influenciado por fatores genéticos, o qual é previsível; e o extrínseco ou fotoenvelhecimento, o qual está associado ao meio ambiente, como a exposição a radiações UV, tabagismo, álcool, nutrição, poluição e outros (MONTAGNER et al., 2009; ADDOR, 2015; AGUIAR et al., 2017; HIRATA et al., 2004)

A qualidade do envelhecimento vai depender de diversificados fatores e da forma como cada um conduz o seu estilo de vida, podendo ser mais ou menos atenuado conforme a qualidade de vida à qual o organismo foi submetido (BENTO, 2015; AGUIAR et al., 2017). Além disso, a entrada na velhice está sujeito a vários aspectos que ultrapassam os limites da mera cronologia, de forma que cada indivíduo reage de forma 
única ao avanço da idade, sendo um processo fisiológico natural desenrolado de diferentes formas (FARINATTI, 2002; TRAMONTINO et al., 2009).

Atualmente um parâmetro importante, que requer maior destaque, seria a influência de uma alimentação inadequada sobre o processo de envelhecimento. Visto que as rotinas mais movimentadas e estressantes levam ao consumo de alimentos ricos em carboidratos e gorduras, comidas rápidas, tipo fast foods, altamente processados (SHIBAO et al., 2011; SCHALKA et al., 2017), assim o excesso de carboidratos na dieta pode induzir a glicação, uma reação não enzimática, que ocorre entre a glicose e as proteínas, gerando os produtos finais da glicação avança (AGEs) (BENTO, 2015). As proteínas com meia vida longa como o colágeno, são as mais susceptíveis à glicação, visto que possui os aminoácidos mais reativos ao processo de glicação, como a arginina e a lisina (ALMEIDA, 2015).

A glicosilação das proteínas, ocorre quando as moléculas de glicose se aderem e entrelaçam-se as moléculas proteicas, fazendo com que estas percam a sua estrutura e por consequência a função (GOLDBEERG et al., 2004; TRAMONTINO et al., 2009), como no caso do colágeno, o qual quando degradado acaba provocando as rugas e perda da elasticidade da pele (TESTON et al., 2010).

Os AGEs são formados após meses ou até mesmo anos de aderência das moléculas de glicose e são relacionados ao estresse oxidativo, com a formação de radicais livres (RL) (TRAMONTINO et al, 2009; AMORIM et al., 2013), mas os RL são moléculas altamente instáveis, com meia-vida extremamente curta e, quimicamente, muito reativas, capazes de causar danos e morte celular (BIANCHI et al., 1999; AMORIM et al., 2013).

A formação de AGEs sob condições fisiológicas ocorre de forma lenta e exerce um papel importante para o processo de envelhecimento, já que compromete as moléculas de meia-vida longa, como o colágeno, que se acumulam no decorrer da vida (AMORIM et al., 2013). Entretanto, sob condições de hiperglicemia, estresse oxidativo, alimentação inadequada, a formação de AGEs é acelerada, assim, por serem estáveis e acumularem nos tecidos, a pele é um dos órgãos de deposito dos AGEs, suas concentrações não retornam à normalidade (SCHALKA et al., 2016; AMORIM et al., 2013).

OS AGEs também são produzidos durante o processo de cozedura dos alimentos, em aplicação de calor ou processamento térmico (URIBARRI et al., 2005). Assim, antes de consumidos, a maioria dos alimentos passam por um processamento térmico, que garante a segurança microbiológica, inativação de algumas enzimas, degradação de substâncias tóxicas e, ainda, o desenvolvimento das substâncias organolépticas, que são responsáveis pelo aroma, cor e sabor, melhorando a palatabilidade do alimento. Estas substâncias são derivadas da Reação de Maillard (BARBOSA et al., 2009; SHIBAO et al., 2011; AMORIM et al., 2013). Os AGEs são gerados nos estágios tardios da RM, tanto em alimentos quanto nos sistemas biológicos (CHUYEN, 2006). A RM que ocorre nos seres vivos é a glicação (SHIBAO et al., 2011; YAMAGISHI et al., 2007).

A RM é o fenômeno ao qual ocorre o escurecimento dos alimentos na medida que são aquecidos, com o prolongamento do processo térmico ou armazenamento ocorre a formação dos AGEs, os quais são 
muito reativos, podendo reagir com resíduos de lisina e arginina, formando compostos estáveis (BARBOSA et al., 2009; YAMAGISHI et al., 2007).

Assim a dieta é considerada uma das principais fontes exógenas de AGEs potencialmente prejudiciais à saúde e relacionadas ao envelhecimento cutâneo, sendo que a formação destes produtos nos alimentos é afetada pela composição em nutrientes, temperatura e pelo método de tratamento térmico aplicado durante o processo de preparação. Além de que os AGEs dietéticos se somam ao pool de AGEs endógenos (BARBOSA et al., 2008). Cerca de $10 \%$ dos AGEs que são ingeridos com a dieta são absorvidos pelo organismo. E da fração absorvida quase $2 / 3$ são armazenados e apenas $1 / 3$ é eliminado pela urina, dentro de aproximadamente 48 horas, isso em indivíduos com a função renal normal (BARBOSA et al., 2008; AMORIM et al., 2013).

Os AGEs estão presentes em alimentos submetidos a qualquer tipo de processamento térmico, como alimentos fritos, assados em churrasqueiras, cozidos em forno convencional ou de micro-ondas, sendo a temperatura o parâmetro crítico para a formação destes compostos. Logo os métodos de cozimento mais brandos, e com alto teor de água, como os ensopados e cozimento a vapor, geram teores menores de AGEs nos alimentos (SHIBAO et al., 2011). As comidas cruas não tem quase nenhuma quantidade de AGEs em sua composição (JUNIOR et al., 2007). O teor de AGEs em peito de frango, por exemplo, varia de 1.100kU/100g - quando cozido - a 4.700kU/100g, quando frito (SHIBAO et al., 2011). Assim os alimentos do grupo dos lipídeos apresentam maior quantidade de AGEs com média de $100+/-19 \mathrm{kU} / \mathrm{g}$, seguido pelas proteínas 43 +/- 7kU/g, os carboidratos contêm os valores mais baixos de AGEs, 3,4 +/- 1.kU/g (GOLDBERG et al., 2005).

Com relação a temperatura de cozimento, duração do tempo de cozimento e presença de umidade, o churrasco $\left(225^{\circ} \mathrm{C}\right)$ e a fritura $\left(177^{\circ} \mathrm{C}\right)$ resultam nos níveis mais elevados de $A G E s$, seguido de assar $\left(177^{\circ} \mathrm{C}\right)$ e ferver $\left(100^{\circ} \mathrm{C}\right)$ (GOLDBERG et al., 2005). Dessa forma a formação de AGEs nos alimentos é potencializada por métodos de preparo que utilizam altas temperaturas e baixa umidade (fritar, assar e grelhar), assim que os lipídeos são os principais contribuintes do conteúdo dietético de AGEs (BARBOSA et al., 2008).

Em contrapartida, uma alimentação adequada e balanceada, pobre em alimentos processados e industrializado (ricos em gorduras e carboidratos), possui compostos com efeito antioxidante e/ou antiglicação, como a vitamina C, vitamina A, vitamina $E$, reduzem a formação de AGEs pelo organismo e são capazes de sequestrar RL com grande eficiência, mantendo a estrutura e função a das fibras de colágeno (BARBOSA et al., 2009; SHIBAO et al., 2011; BIANCHI et al., 1999).

Os antioxidantes são qualquer substância que, presente em baixas concentrações, em relação ao substrato oxidável, atrasa ou inibe a oxidação do substrato de maneira eficaz. São capazes de interceptar radicais livres gerados pelo metabolismo celular ou por fontes exógenas, de forma a impedir o ataque sobre os lipídeos e os aminoácidos das proteínas, evitando a formação de lesões e perda da integridade celular (BIANCHI et al., 1999). Eles são aplicados topicamente exercendo, também, os seus benefícios, pois oferecem proteção contra RL prejudiciais a pele, permitindo um envelhecimento natural e um rejuvenescimento da pele (FARRIS, 2005). 


\section{CONCLUSÃO}

As informações apresentadas nesta revisão, apontam para ao fato de que os AGES presentes na alimentação (dieta), provindos da RM e os produzidos endogenamente a partir da glicação, constituem importantes determinantes do pool endógeno final desses compostos no organismo. Além de que a modificação dos hábitos alimentares da população induzem ao envelhecimento precoce da pele, devido ao maior consumo de alimentos ricos em açúcares e gorduras, onde há uma maior preferência ao consumo de alimentos processados e industrializados, por serem de mais fácil acesso, devido ao ritmo de vida acelerado. Desse modo, visto a relação direta do consumo desse alimentos com a formação do AGEs, é preciso que se reduza o consumo destes alimentos, para assim evitar o envelhecimento precoce.

Como a forma de processamento do alimento influencia na formação dos AGEs devido a RM, onde o processamento térmico em altas temperaturas $(>170 \circ \mathrm{C}$ ) potencializa a formação dos AGEs (frituras, churrascos e grelhados), as alterações como adotar o cozimento a temperaturas próximas de $100^{\circ} \mathrm{C}$, em presença de água (ensopados e alimentos cozidos a vapor), por curto período de tempo, como forma de preparo do alimento podem ter uma repercussão significativa na redução dos AGEs exógenos.

Sabendo que os AGEs podem gerar os RL, devido ao estresse oxidativo, deve-se incluir na dieta o consumo de alimentos in natura, além de aumentar o consumo de frutas, verdura e legumes, ricos em vitaminas antioxidantes (vitaminas A, C e E), que auxiliam na redução dos AGEs e RL no organismo, levando a reconstrução celular, rejuvenescimento da pele, manutenção dos tecidos e melhor funcionamento do organismo.

\section{REFERÊNCIAS}

ADDOR, F. A. S.. Influence of a nutitional supplemente containing collagem peptides on the properties of the dermis. Surgical \& Cosmetic Dermatology, v.7, n.2, p.116121, 2015. DOI: http://dx.doi.org/10.5935/scd1984$\underline{8773.201572636}$

AGUIAR, R. V. S. C..; OLIVEIRA, C.; BARELLI, N.; MELO, B.; GONÇALVES, T.; FEITOSA, G. P. V.. Fotoenvelhecimento nos diferentes grupo étnicos. Revista de Iniciação Cientifica, Saúde e Bem-estar, v.6, n.5, p.19-30, 2017.

ALMEIDA, M. E. S.. Papel da Glicação do colágeno I e da alta concentração de glicose sobre a migração de fibroblastos. Tese (Doutorado em Biologia Celular e Tecidual) - Instituto de Ciências Biomédicas, São Paulo, 2015.

AMORIM, N. O. B.; LISBOA, H. R. K.; SIQUEIRA, L. O.. Glicação não enzimática de proteínas na gênese da nefropatia diabética. Revista HCPA, v.33, n.2, p.135-141, 2013.

BARBOSA, J. H. P.; SOUZA, I. T.; SANTANA, A. E. G.; GOULART, M. O. F.. A determinação dos produtos avançados de glicação (AGES) e de lipoxidação (ALES) em alimentos e sistemas biológicos: Avanços, desafios e perspectivas. Química Nova, v.39, n.5, p.608-620, 2016. DOI: http://dx.doi.org/10.5935/0100-4042.20160048

BARBOSA, J. H.P.; OLIVEIRA, S. L.; SEARA, L. T.. O papel dos produtos finais da Glicação avançada (AGEs) no desencadeamento das complicações vasculares do diabetes. Arquivos Brasileiros de Endocrinologia \& Metabologia, v.52, n.6, p.940-950, 2008.

BARBOSA, J. H.P.; OLIVEIRA, S. L.; SEARA, L. T.. Produtos da Glicação avançada dietéticos e as complicações crônicas do diabetes. Revista de Nutrição, Campinas, v.22, n.1, p.113124, 2009.

BATISTELA, M. A.; CHORILLI, M.; LEONARDI, G. R.. Abordagens no estudo do envelhecimento cutâneo em diferentes etnias. Revista Brasileira de Farmácia, v.88, n.2, p.59-62, 2007.

BENTO, B. S.. Fotoenvelhecimento cutâneo: processo/produtos. Dissertação (Mestrado) - Instituto Superior de Ciências da Saúde Egas Moniz, 2015.

BIANCHI, M. L. P.; ANTUNES, L. M. G.. Radicais livres e os principais antioxidantes da dieta. Revista de Nutrição, Campinas, v.12, n.2, p.123-130, 1999.

CHUYEN, N. V.. Toxicity of the AGEs generated from the Maillard reaction: On relationship of food-AGEs and biological-AGEs. Molecular Nutrition \& Food Research, v.50, n.12, p.1140-1149, 2006. DOI: https://10.1002/mnfr.200600144 
FARINATTI, P. T. V.. Teorias biológicas do envelhecimento: do genético ao estocástico. Revista Brasileira de Medicina do Esporte, v.8, n.4, p.129-138, 2002.

FARRIS, P. K.. Topical vitamin C: a useful agente for treating photoaging and other dermatologic conditions.

Dermatologic Surgery, v.31, p.814-818, 2005. DOI: https://doi.org/10.1111/j.1524-4725.2005.31725

GOLDBERG, T.; CAI, W.; PEPPA, M.; DARDAINE, V.; BALIGA, B. S.; URIBARRI, J.; VLASSARA, H.. Advanced glycoxidation end products in commonly consumed foods. Journal of the American Dietetic Association, v. 104, n. 8, p. 1287-1291, 2004. DOI: https://10.1016/j.jada.2004.05.214

HIRATA, L. L.; SATO, M. E. O.; SANTOS, C. A. M.. Radicais Livres e o envelhecimento cutâneo. Acta Farmaceutica Bonaerense, v.23, n.3, p.418-424, 2004.

LAPOLLA, A.; FEDELE, D.; REINATO, R.; ARICO, N. C.; SERAGLIA, R.; TRALDI, P.; MAROTTS, E.; TONANI, R.. Enzymatic digestion and mass spectrometry in the study of advanced glycation end products/peptides. Journal of the American Society for Mass Spectrometry, v.15, p.496-509, 2004. DOI: https://doi.org/10.1016/j.jasms.2003.11.014

MONTAGNER, S.; COSTA, A.. Bases Moleculares do fotoenvelhecimento. Anais Brasileiros de Dermatologia, v.84, n.3, p. 263-269, 2009. DOI: https://10.1590/S036505962009000300008

SCHALKA, S.; MAGALHÃES, W. V.; CAZERTA, C.; SHITARA, D.; SUFI, B. S.; QUADROS, A.. Composto nutracêutico aumenta a síntese de colágeno, elastina e ácido hialurônico. Surgical \& Cosmetic Dermatology, v.9, n.1, p. 46-50, 2017. DOI: http://dx.doi.org/10.5935/scd1984-8773.201791875
SCHALKA, S.; BECHELLI, L.; BOMBARDA, P. C. P.; ABREU, F. F.. Uma nova proposta para avaliação de cosmecêutico antioxidante no tratamento da pele afetada pelos efeitos da vida urbana. Surgical \& Cosmetic Dermatology, v.8, n.1, p. 46-54, 2016. DOI: http://dx.doi.org/10.5935/scd1984$\underline{8773.201681753}$

SHIBAO, J.; BASTOS, D. H. M.. Produtos da reação de Maillard em alimentos: implicações para a saúde. Revista de Nutrição, Campinas, v.24, n.6, p.895-904, 2011.

TESTON, A. P.; NARDINO, D.; PIVATO, L.. Envelhecimento cutâneo: Teoria dos radicais livres e tratamento visando a prevenção e rejuvenescimento. UNINGÁ Review, v.1, p.7184, 2010.

TRAMONTINO, V. S.; NUÑEZ, J. M. C.; TAKAHASHI, J. M. F. K.; SANTOS-DAROZ, C. B.; RIZZATTI-BARBOSA, C. M.. Nutrição para Idosos. Revista de Odontologia da Universidade de São Paulo, v.21, n.3, p.258-267, 2009.

URIBARRI, J.; CAI, W.; SANDU, O.; PEPPA, M.; GOLDBERG, T.; VLASSARA, H.. Diet-derived advanced glycation end products are major contributors to the body's AGE pool and induce inflammation in healthy subjects. Annals of the New York Academy of Sciences, v.1043, p.461-466, 2005. DOI: https://doi.org/10.1196/annals.1333.052

YAMAGISHI, S.; UEDA, S.; OKUDA, S.. Food-derived advanced glycation end products (AGEs): A novel therapeutic target for various disorders. Current Pharmaceutical Design, v.13, n.27, p.2832-2836, 2007. DOI: https://10.2174/138161207781757051

A CBPC - Companhia Brasileira de Produção Científica (CNPJ: 11.221.422/0001-03) detém os direitos materiais desta publicação. Os direitos referem-se à publicação do trabalho em qualquer parte do mundo, incluindo os direitos às renovações, expansões e disseminações da contribuição, bem como outros direitos subsidiários. Todos os trabalhos publicados eletronicamente poderão posteriormente ser publicados em coletâneas impressas sob coordenação da Sustenere Publishing, da Companhia Brasileira de Produção Científica e seus parceiros autorizados. Os (as) autores (as) preservam os direitos autorais, mas não têm permissão para a publicação da contribuição em outro meio, impresso ou digital, em português ou em tradução. 